\title{
Cell biology befriends soft matter physics
}

\author{
Phase separation creates complex condensates in eukaryotic cells. To study these mysterious droplets, many \\ disciplines come together.
}

\section{Vivien Marx}

n n many cartoons, eukaryotic cells have a tidy look. A taut membrane surrounds a placid, often pastel-hued lake with well-circumscribed organelles such as ribosomes and a few bits and bobs. It's a helpful simplification of the jam-packed cytoplasm. In some areas, there's even more intense milling about ${ }^{1-4}$. To get a sense of these milieus, head to the kitchen with Tony Hyman of the Max Planck Institute of Molecular Cell Biology and Genetics and Christoph Weber and Frank Jülicher of the Max Planck Institute for the Physics of

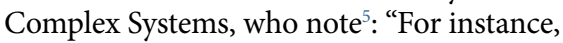
when you make vinaigrette and leave it, you come back annoyed to find that the oil and vinegar have demixed into two different phases: an oil phase and a vinegar phase." Just as oil and vinegar can separate into two stable phases, such liquid-liquid phase separation can take place in the cytoplasm. This creates condensates, which are malleable, liquid-like areas where proteins, peptides and RNAs congregate that might otherwise be on opposite sides of Lake Cytoplasm. P-bodies and stress granules are some of the condensates in the cytoplasm. Nucleoli and Cajal bodies are some of those in the nucleus. Unlike organelles, condensates have no membrane, but there is a "non-membrane boundary," says Hyman, with, for example, density and concentration unlike those of the surrounding cytoplasm or nucleoplasm. Condensates are a little like flash mobs, says Hyman. When the music is on, people come together, and when the music stops, they disperse. Condensates are dense locales. A eukaryotic cell typically contains billions of proteins. A condensate might be one to two micrometers in diameter and hold around one million proteins. It's akin, he says, to situating the world's population in greater Los Angeles. And everyone in this megalopolis stays on the move.

Components in a condensate tumble around one another, touch briefly and move on, which leads to a dynamic of interactions, says Princeton University researcher Clifford Brangwynne. A wide range of biochemical activities take place in condensates, such as chromatin organization or stress responses. Early papers in the condensate field were more qualitative and descriptive, but that is

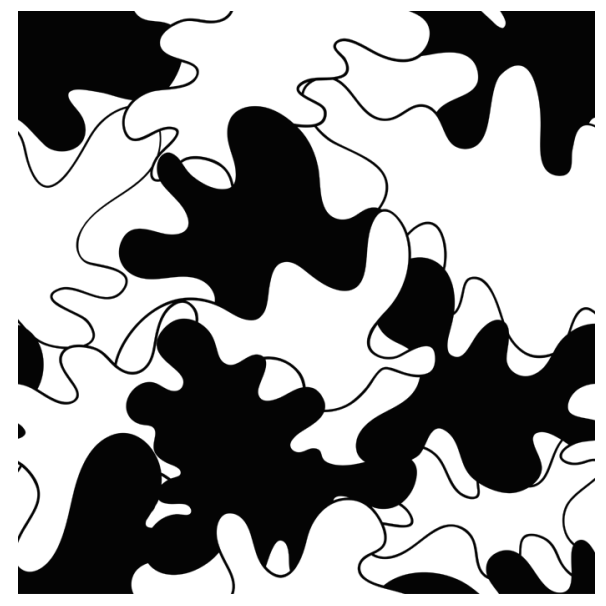

Eukaryotic cells contain mysterious, malleable, liquid-like condensates in which there is intense activity. Credit: Y. Drozdova/Alamy Stock Vector.

giving way to rigorous approaches and tools to study "native biological condensates" and manipulate them, he says. "Methods are still pretty underdeveloped in this field," he says. Given that molecular forces and biophysical factors drive condensate formation and maintenance, advances need to draw on several disciplines. Cell and molecular biologists, proteomics labs and those in polymer physics are intrigued by condensates.

Brangwynne, then a postdoctoral fellow in the Hyman lab, discovered that $\mathrm{P}$ granules phase-separate in the posterior of the embryo as part of germ cell development in Caenorhabditis elegans ${ }^{1}$. During a research stint at the Marine Biological Laboratory in Woods Hole, Massachusetts, and using quantitative particle tracking, he and one of the students in the physiology course tagged $\mathrm{P}$ granules and showed how they move, condense and coalesce to bring RNAs and proteins together. Hyman, Jülicher and colleagues were excited about the finding, says Brangwynne. "Such phase transitions may represent a fundamental physicochemical mechanism for structuring the cytoplasm," the authors noted. P granules may look granular, but they are actually phase-separated liquid droplets. What is known, says Hyman, is that the cell's proteins interact and 'sample' one another.
When the right contacts are made, phase separation occurs.

\section{Methods multitude}

In the young condensate field, Amy Gladfelter of the University of North Carolina at Chapel Hill says her lab intertwines molecular-biology-based approaches, modeling, and in vitro reconstitution experiments using simplified systems. "You can't build models that have all the parameters that exist in a cell," she says. But researchers need to consider that in vitro work or overexpressing proteins will not do complete justice to condensates in situ. "Anything will phase separate in a test tube," she says, highlighting experiments in which crowding agents such as polyethylene glycol are used to induce phase separation. Another widely used method involves measurement of fluorescence recovery after photobleaching. Such FRAP-ing, "that's just not telling you that much." Better measurements are needed to, for example, characterize viscosity in a condensate, she says. New types of density probes will be useful, as will dyes that fluoresce to reflect how crowded a condensate is. Her lab applies rotor dyes to assess how much 'tumbling' is taking place in a condensate. One organism Gladfelter studies is the fungus Ashbya, which has multinucleated cells, as do other organisms and human muscle cells, too. In her Woods Hole summer lab, she began collaborating on condensates with Brangwynne, Hyman, Michael Rosen of the University of Texas Southwestern Medical Center and others.

In Ashbya's non-membrane-bound areas, she came across Whi3, an RNA-binding

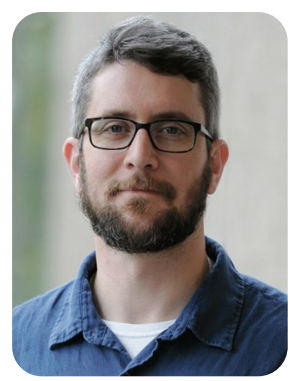

"If we're trying to test quantitative biophysical models, let's use quantitative biophysical approaches to do it," says Clifford Brangwynne. Credit: John D. \& Catherine T. MacArthur Foundation 
protein. Her team in collaboration with Sua Myong, now at Johns Hopkins University, used techniques such as single-molecule fluorescence resonance energy transfer (FRET) and found that Whi3 binding changes the conformational dynamics of the RNAs it targets. RNAs and the RNA-protein interactions shape condensate formation and maintenance and give condensates distinct identities. Condensates will differ in their Whi3 levels and location. Some condensates form at the cell tips where growth is taking place. Gladfelter explores molecular mechanisms of condensate dynamics to understand how sequestering a biochemical reaction in a condensate has advantages for the cell. Since condensates are so dynamic, classic structural biology approaches to studying proteins and protein complexes don't address all of a condensate's complexity, says Hyman. Mass spectrometry can help labs establish the metabolites in a condensate. And when condensates can be purified and they stay intact, says Gladfelter, mass spec helps to identify proteins, which has worked with stress granules, for example. For the RNAs, labs can use RNA sequencing. For the condensates she studies, however, "we have not been able to purify the condensates and do that." Nuclear magnetic resonance spectroscopy (NMR) has been applied to some proteins relevant to phase separation. She points to the wealth of tools from the Brangwynne lab and says, "I personally don't think any one thing is sufficient." The method optoDroplet ${ }^{6}$, developed in the Brangwynne lab, uses light to trigger protein association and selectively control a condensate. Researchers can look at intrinsically disordered proteins in condensates, initiate intracellular protein clustering and study phase separation.

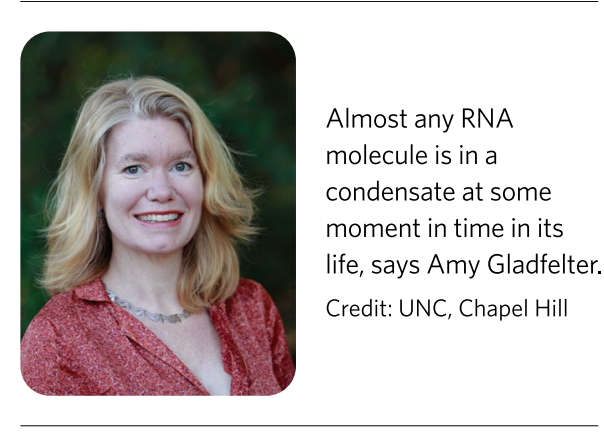

CasDrop ${ }^{7}$ from the Brangwynne lab is an optogenetic approach to address genes of choice in a condensate. One might, says Gladfelter, use it to place a condensate in the nucleus. To study multivalency of proteins in a condensate, one might choose Corelet $^{8}$, also from the Brangwynne lab.

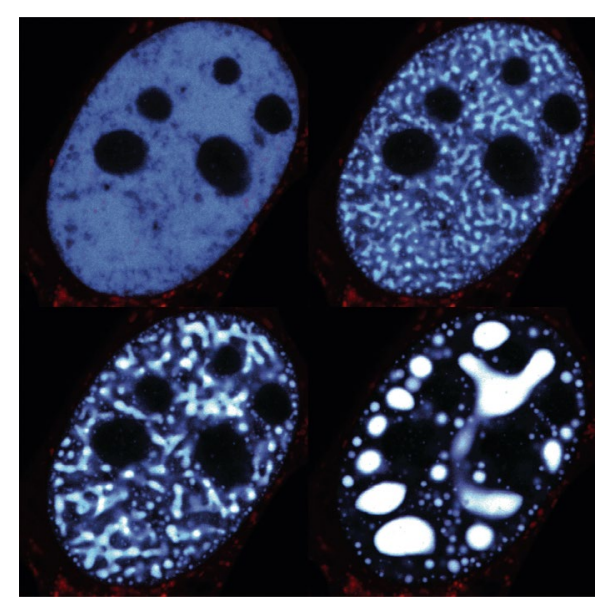

Optogenetics methods can be used to study the biophysics of phase separation. Shown here, the nucleus of a living human cell at different times after optogenetic activation with blue light.

Credit: D. Bracha, C. Brangwynne, Princeton U.

It's a way to get a quantitative readout of the intrinsically disordered proteins that might be driving phase separation in cells. Brangwynne is particularly fond of Corelet because it helps query how oligomerized an environment has to be for a condensate to form. "It lets us map these phase diagrams," he says. Corelet involves a self-assembled GFP-tagged particle, with a 24-mer ferritin core in which each core is fused to an optogenetic domain. As the particles 'touch' proteins with internally disordered regions, more or fewer proteins attach, depending on conditions. Corelet is like having an "entropy knob" on cells, says Brangwynne. Just as temperature changes a system's entropy, so condensates can have altered entropy levels. Probing the interactions of proteins with corelets, researchers find out how much these components "want to stay together," he says. "That's a technique I really like for that reason." Recently, his lab developed a way to extract thermodynamic parameters from microscopy images of condensates 9 . To do so, the team applied Corelet to manipulate proteins in several condensates.

\section{Stickers and spacers}

By iterating between experiment and computational modeling, Rohit Pappu and his team at Washington University developed the 'stickers and spacers' model, a framework that enables one to describe and predict condensate formation on the basis of protein attributes ${ }^{4}$. Intrinsically disordered regions of proteins have long been considered drivers of condensate formation and maintenance. The Pappu lab, the Rosen lab and others have shown that folded proteins play a role, too, says Pappu. Discussions over foldedness versus intrinsic disorder have given way to the concept of multivalency, says Pappu, which the model represents. Adapting concepts from polymer science, the model considers segments along a protein's chain of amino acids to be more or less prone to interactions: some behave like 'stickers', others as 'spacers'. "The balancing of sticker-to-spacer ratio becomes quite important," he says. With only stickers, a protein can end up a goopy mess, which can be a hallmark of disease. Phase separation, says Pappu, may play a role in Huntington's disease. A mutation in the huntingtin gene leads to a polyglutamine repeat that contributes to fibrillary tangles in cells. Working on huntingtin led him to condensates, says Pappu. Using online resources, scientists can find out how sticky individual residues are, but these resources lack context for the particular sequence one is looking at, says Pappu. This motivates him to contribute to the community's efforts to build "a rigorous grammar" of condensates to help predict, from sequence, how proteins will interact.

This prediction is informed by experiments such as single-molecule FRET measurements that capture resonance transfer efficiencies in disordered proteins. Pappu's then postdoctoral fellow Kiersten Ruff worked out how to give FRET measurements a molecular context with all-atom simulations and machine-learning approaches to characterize protein ensembles. Calculated FRET efficiencies are calibrated with experimental ones. What results is calculated conformational heterogeneity of proteins in condensates. They have a general "tadpole architecture," he says, with a more globular head and a tail where the spacers are. In the protein huntingtin, the glutamines are stickers, but in a different protein, fused in sarcoma (FUS), glutamines and asparagines are not as potent stickers. "Who is a spacer and who is be sticker will become context dependent," says Pappu. Using this model, the team has built a computational pipeline with which people can delineate their stickers and spacers. It takes into account post-translational modifications, which modify the strengths of the stickers and spacers. Experimental measurements feed into this computational pipeline, as do scattering, single-molecule FRET, NMR and hydrogen deuterium exchange data. "The prospecting happens with computation," he says. When researchers apply this framework to computationally identify stickers and spacers, it helps them decide which protein region to query experimentally. They can map out condensate changes using calculated phase diagrams. Altering stickers and spacers changes valency and thus 
shapes a dynamic in a condensate. "This is a field where you cannot become a one-trick pony," says Pappu. Given the diversity of challenges, one needs a "kitchen sink" of methods. Yet an individual lab might not have the time, resources and skills to readily use, for example, small-angle X-ray scattering (SAXS), NMR and hydrogendeuterium exchange. "You don't want to say to every cell biologist: you have to do NMR and SAXS and single-molecule FRET," he says. Perhaps, he says, this predictive computational engine can lead to 'codifiable rules' to target experiments and reveal the molecular grammar of condensates.

Stickers and spacers is a useful representation and a way to reduce the complexity of factors that shape a condensate and prepare how to test them experimentally, says Hyman. A sticker, says Brangwynne, has affinity for one type of molecule and a different affinity for another. "You put them all together and ask: what does the network of interactions look like?" This is one way condensate research draws on approaches in polymer physics.

\section{RNAs, too}

As condensate research emerged, RNAs were rather neglected, says Gladfelter, but that is changing ${ }^{10,11}$. "It's pretty fair game to say that almost any RNA molecule is in a condensate at some moment in time in its life." Proteins interact with one another and with RNAs, which renders condensate dynamics even more complex, says Hyman. Getting at this richness takes input from different fields: molecular and cell biology, chemistry, physical chemistry and polymer physics, he says. Whereas labs working in physics and polymer physics are happy with the concept of 'states' that a material can have, molecular

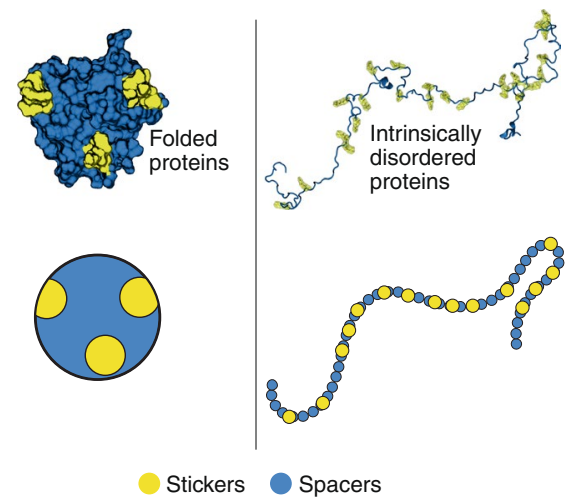

The Pappu lab developed the 'stickers and spacers' model for computationally predicting condensates on the basis of protein attributes. Credit: Pappu Lab, Washington U. Reproduced with permission from Annu. Rev. Biophys.

49 (c) 2020 Annual Reviews. biologists focus on amino acids as the level that evolutionary forces act upon. There may be a 'state of the polymer' as a function of individual amino acids. "We need to try to understand the range of protein dynamics in a particular context," says Hyman. New types of FRET measurement may help, as might label-free imaging techniques such as the light-scattering process that underlies Brillouin microscopy and other approaches used to study the elasticity of condensed matter.

What is emerging is that in the interactions between proteins and RNAs, says Brangwynne, sometimes disordered regions in proteins play more modulatory roles. In his work, he draws on network-based methods and concepts from soft matter physics. "It's about connectivity," he says. "The field has been pretty protein-centric for a while," he says. "We're to blame for that, and others as well," he says. RNA can modulate a condensate's protein-protein network, "but RNA can play an important scaffolding role." Ultimately one needs a sufficient number of interactions, he says, which is where the concept of valency comes in.

\section{Applied condensates}

Condensates might propel research on neurodegeneration and cancer. Hyman and Whitehead Institute researcher Rick Young cofounded Dewpoint Therapeutics, a company based in Boston and Dresden. In November 2019, Bayer invested $\$ 100$ million in the company. In a statement, Joerg Moeller, who directs research and development in Bayer's Pharmaceuticals Division, said new analytic tools and a growing understanding of biomolecular condensates could shed light on cellular functions that drug development scientists have not previously considered and help to identify pharmacological targets. Hyman believes condensates can be used to explore a drug candidate's cell biological mechanisms, to characterize the thermodynamics and kinetics of the condensate and the actions of a drug whose target might be in a condensate.

For Edward Lemke, condensates were part of the solution to a problem he and his team at the European Molecular Biology Laboratory, Johannes Gutenberg University and Institute of Molecular Biology Mainz were trying to solve: how to precisely position non-canonical amino acids in engineered proteins $^{12}$. They used condensates to bring together, in a eukaryotic cell, the components of an 'orthogonally translating synthetic designer organelle'. The team targets a particular tRNA and mRNA to an assembly that also contains proteins, such as FUS and kinesin motor proteins. His motivation, says

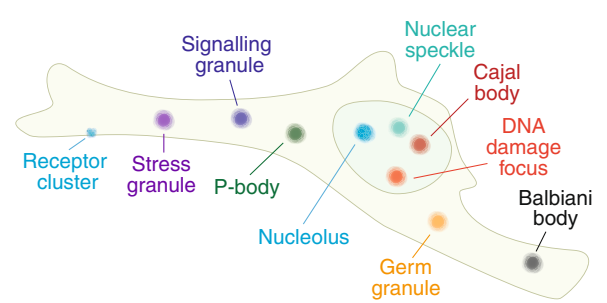

The cytoplasm and nucleoplasm of eukaryotic cells reveal many types of condensates that sequester a variety of biochemical reactions. Credit: Pappu Lab, Washington U. Reproduced with permission from Annu. Rev. Biophys. 49 (c) 2020 Annual Reviews.

Lemke, is fueled by an interest in biophysics and the need for useful in-cell fluorescent labels for studying intrinsically disordered proteins. The contrast in imaging readouts can be faint in experiments that involve genetic code expansion, in which synthetic biologists expand beyond the standard protein repertoire of 20 amino acids. Cells do not readily use non-natural amino acids, and off-target effects result. "It's just the tool that works really well for chemists, but it really doesn't work that well for biologists yet," says Lemke. It's challenging to encapsulate this different and complex translation. That's when it occurred to him to use phase separation to do so. He and his team believe the system is scalable. They used FUS, a well-studied protein that always phase separates, and then they engineered a binding site for their intended RNA. The system is readily reproducible, he says, and it recruits needed components into the system. A lack of membrane means ribosomes can "scoot over." Although a ribosome or a tRNA might leave the designated area, the concentration gradient prevents that for the most part. "Basically our organelle is a heat-sink for our tRNA," he says. It's where the tRNA's codon is translated differently than outside this area. The scientists made many binding sites within a droplet for the tRNA by actively targeting its synthetase, the enzyme that catalyzes the reaction to charge a tRNA with a specific amino acid. The approach shows that translation likely involves around 100 factors, which travel into and then out of the condensate. It shows "you can basically switch how translation works, simply by phase separating," he says. "Chemistry inside the phase-separated organelle can be very, very, very different from the cytoplasm."

The approach to expand the genetic lexicon beyond 20 amino acids was developed by Peter Schultz at Scripps Research and University of California, Berkeley and his team, and it's a powerful synthetic biology tool, says Lemke. In 


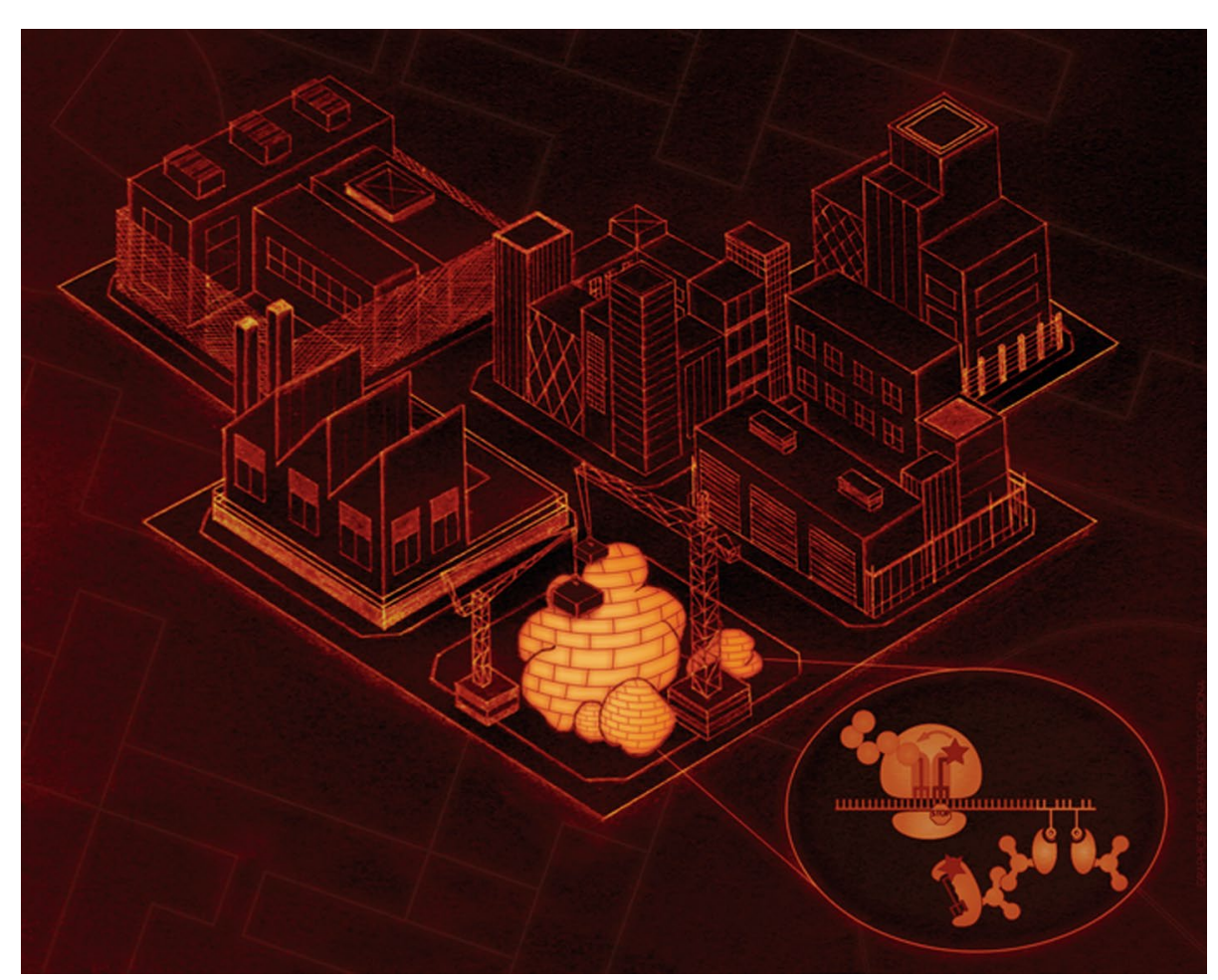

The cellular factory makes proteins with canonical amino acids. Within a condensate, the Lemke lab built an artificial organelle to also make proteins with noncanonical amino acids. Credit: E. Lemke, G. Estrada Girona, IMB/U Mainz/EMBL

this experiment, Lemke's lab tweaked the approach to work well in eukaryotes, where codon specificity has been a challenge. "It's always easier to engineer an organelle than a eukaryotic host," he says. "We decoupled host engineering from organelle engineering." The approach opens up many possibilities for work in eukaryotic cells, says Lemke. More label colors are needed to track proteins, and off-target issues need to be avoided. The artificial organelle should be smaller and less toxic to the cell, and he has ideas how to make that happen. "Then I think the actual cell biologist will appreciate the technology that we have," he says. Lemke launched a startup, Araxa Biosciences, focused on protein engineering tools for antibody therapeutics, where he is chief scientific officer. The company has an investor and it licensed the organelle technology from his lab. Perhaps there is no limit to the complexity of the tasks an organelle can pull off. Translation is not just a simple enzymatic reaction, he says. Perhaps, he speculates, the method of sequestering reactions in condensates has been a driving force in evolution in eukaryotic cells. Membrane-bound organelles likely emerged when cytoplasmic housekeeping needed more selectivity. For example, the lysosome, the cell's waste disposal system, has advantages when it is encapsulated by a membrane. "That's probably, I would guess, how the nucleus must have evolved," he says.

\section{Beyond a Tower of Babel}

In a recent paper $^{13}$, Robert Tjian at the University of California, Berkeley and colleagues point out that various mechanisms can lead to condensates and that the tests commonly used to probe liquid-liquid phase separation (LLPS) "are insufficient to rule out other mechanistic interpretations." Among other aspects, the team notes, "we urge the application of more stringent criteria and more appropriate experimental approaches to understand the functional role of LLPS condensates in cellular organization."

Although some in the condensate community have reacted with irritation, Hyman calls the paper "perfectly legitimate" in its advice for caution. "He asks some very good questions," says Hyman, such as what confers size stability on condensates in the nucleus, which is not trivial from a physics point of view. Hyman says he reminds his lab that "the last thing you need is cheerleaders." Rather, it's naysayers who push researchers to keep devising experiments to drive insight to answer the question. He and Brangwynne saw condensates for the first time in 2009. Over time, "you begin to revise things you didn't quite understand in the beginning." Brangwynne agrees that the Tjian team raises some valid points. There have been some "handwavy" papers that only purport but do not test the validity of a quantitative biophysical framework. "If we're trying to test quantitative biophysical models, let's use quantitative biophysical approaches to do it." It's insufficient, for example, to state that one has detected a "diffraction-limited spot, so it must be phase separated." The condensate field needs collaboration across disciplinary divides, which is never easy, says Hyman. He has built a group with team leaders from different disciplines. $\mathrm{He}$ notices that researchers might use the same words that have different meanings and that different words can mean the same thing in different fields. "Right now it's a little bit of a Tower of Babel," he says. Over time and with successful collaborations, this will change. Lemke's group includes physicists, chemists, biologists and bioengineers. Half of his group does biophysics and the other half chemical biology and synthetic biology. Some members of the Gladfelter lab joined the lab as mathematical modelers, others focus on in vitro work for the biophysical analysis of phase separation and others do in vivo experiments, such as confocal image analysis in live cells. She trains them each to be involved in at least two of those areas. One of her students, an applied mathematician who built a condensate model, has been trying his hand at experiments and doing well, she says. The field needs better ways to explore the compositional complexity of condensates in situ. "There's a lot of challenges ahead that necessitate technical development," she says. Concerning the fact that labs are using many different methods to characterize condensates, "I think that's actually one of the cool things about the field."

Vivien Marx ${ }^{凶}$

Technology editor for Nature Methods.

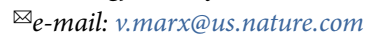

Published online: 20 May 2020

https://doi.org/10.1038/s41592-020-0855-3

References

1. Brangwynne, C. P. et al. Science 324, 1729-1732 (2009).

2. Boeynaems, S. et al. Trends Cell Biol. 28, 420-435 (2018).

3. Alberti, S., Gladfelter, A. \& Mittag, T. Cell 176, 419-434 (2019).

4. Choi, J.-M., Holehouse, A. S. \& Pappu, R. V. Annu. Rev. Biophys. 49, 107-133 (2020).

5. Hyman, A. A., Weber, C. A. \& Jülicher, F. Annu. Rev. Cell Dev. Biol. 30, 39-58 (2014).

6. Shin, Y. et al. Cell 168 159-171.el4 (2017).

7. Shin, Y. et al. Cell 175 1481-1491.e13 (2018).

8. Bracha, D. et al. Cell 175 1467-1480.e13 (2018).

9. Riback, J. A. et al. Nature https://doi.org/10.1038/s41586-0202256-2 (2020).

10. Langdon, E. M. et al. Science 360, $922-927$ (2018).

11. Deniz, A. A. Cell 181, 228-230 (2020).

12. Reinkemeier, C. D. et al. Science 363, 1415 (2019).

13. McSwiggen, D. T., Mir, M., Darzacq, X. \& Tjian, R. Genes Dev. 33, 1619-1634 (2019). 